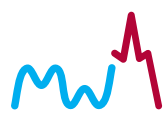

Microwave Engineering

Ulm University

\title{
A Dielectric Lens Antenna Fed by a Flexible Dielectric Waveguide At $160 \mathrm{GHz}$
}

Martin Geiger, Martin Hitzler, Johannes Iberle, Christian Waldschmidt 


\title{
A Dielectric Lens Antenna Fed by a Flexible Dielectric Waveguide At $160 \mathrm{GHz}$
}

\author{
Martin Geiger, Martin Hitzler, Johannes Iberle, Christian Waldschmidt \\ Institute of Microwave Engineering \\ Ulm University \\ 89081 Ulm, Germany \\ martin-2.geiger@uni-ulm.de
}

\begin{abstract}
Flexible antennas in radar applications enable the user to go around obstacles or detect targets at hidden places. In this paper, two elliptical lenses of different size made of high density polyethylene and stacked on a flexible dielectric waveguide are designed and measured from $140 \mathrm{GHz}$ to $180 \mathrm{GHz}$. The feeding dielectric waveguide and the mode transition from metallic waveguide to dielectric waveguide was investigated with full wave simulations. The elliptical lenses were designed with a geometrical optics approach. The realized antennas have a gain larger than $24 \mathrm{dBi}$ and $27 \mathrm{dBi}$ and a maximum side lobe level below $-15.8 \mathrm{~dB}$.
\end{abstract}

Index Terms-flexible lens antenna, dielectric waveguide, rectangular waveguide, mode transformer.

\section{INTRODUCTION}

Dielectric waveguides have been known for a long time [1] and they are used in different applications like in flexible measurement setups for field scanning [2]. The flexibility in bending, low attenuation, and low costs in fabrication make the dielectric waveguide suited for the submillimetre wave range with some advantages over the rectangular waveguide.

To transmit a signal into free space, the dielectric waveguide is tapered at the end to become a dielectric rod antenna [3], [4]. These antennas are broadband but limited to a low gain. For this reason, they are usually used as feed for a lens with large gain [5]. The disadvantage of the lens is the larger antenna size and also the fixed position.

Generally, the antenna gain can also be improved with an elliptical or hemielliptical lens directly mounted on low gain antennas, like patch antennas [6] or open waveguides [7]. With such an elliptical lens directly placed on a dielectric waveguide, the gain can be considerably improved compared to a dielectric rod antenna and the waveguide's flexibility is not lost. Furthermore, the size is very small. These antennas can be used in systems with a fixed transceiver position, where a flexible high gain antenna is required, like in radar systems with a sniffer probe. Compared to a conventional system, only the dielectric waveguide with lens has to be moved instead of the complete RF part.

In this paper, such a flexible lens antenna made of high density polyethylene (HDPE) $\left(\varepsilon_{r}=2.25, \tan \delta=3.1 \cdot 10^{-4}\right)[8]$ with a transition from rectangular waveguide to dielectric waveguide from $140 \mathrm{GHz}$ to $180 \mathrm{GHz}$ is presented. Firstly the design of the antenna and the feeding structure is explained,

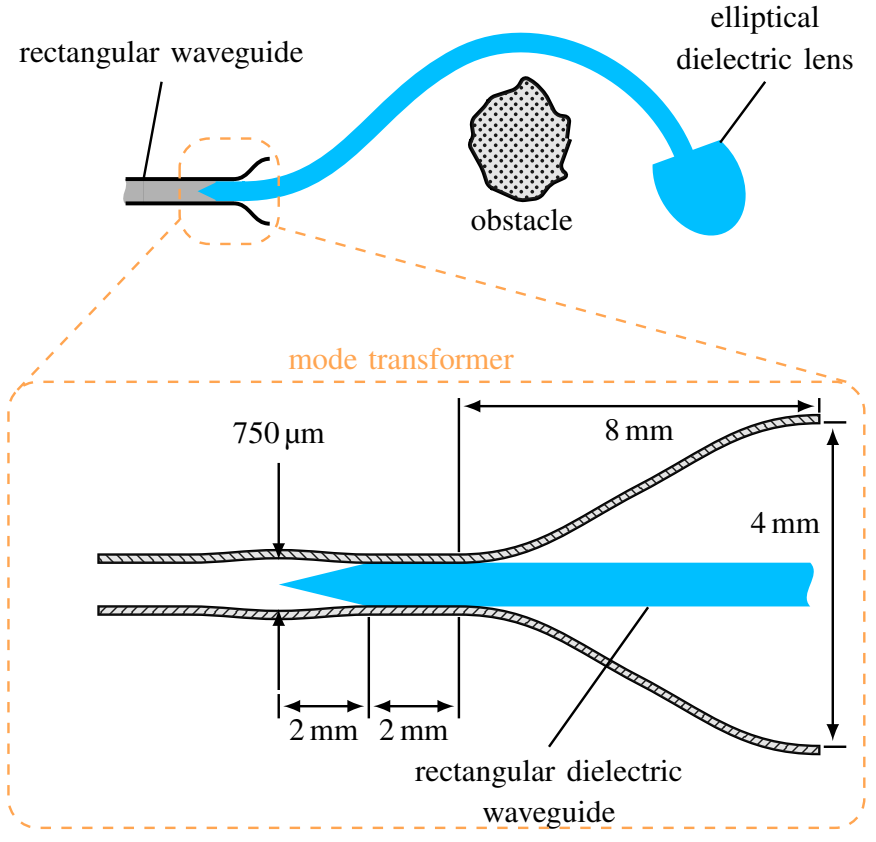

Fig. 1: Schematic view of the elliptical dielectric lens antenna with dielectric feeding line and mode transformer.

afterwards the fabrication process, the complete setup and the measurement results are described.

\section{Antenna FeEding}

The feeding structure for the elliptical lens antenna consists of two parts. The component that is connected to the antenna is a rectangular dielectric waveguide and the second component is a mode transformer from rectangular to dielectric waveguide.

To determine the shape and size of the dielectric waveguide, numerical investigations are necessary, since the flexibility and the losses depend on the cross section. For small dielectric waveguides the dielectric losses are low, because most of the field is guided outside of the waveguide and the effective dielectric constant is small. At discontinuities like bends, where the radius is a measure for the flexibility, a part of the wave's power is radiated into free space. The radiated power decreases with increasing waveguide size [9]. The 


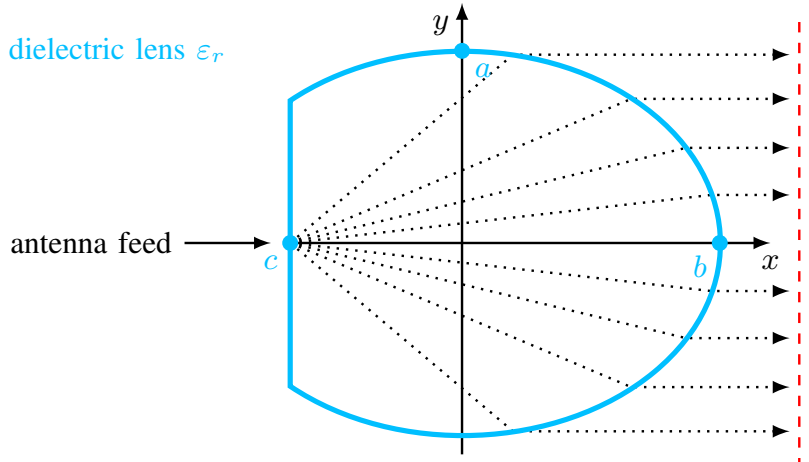

Fig. 2: Schematic view of the elliptical lens with optical paths.

dielectric waveguide feeding also influences its dimensions. A rectangular waveguide can be used as a feeding structure, as the fundamental mode in the rectangular dielectric waveguide is the hybrid $\mathrm{HE}_{11}$ mode, whose field distribution is very similar to the $\mathrm{TE}_{10}$ mode in the rectangular waveguide [10]. Therefore, the chosen dimensions are the WR5 dimensions $(648 \mu \mathrm{m} \times 1295 \mu \mathrm{m})$ and the dielectric waveguide is fed with a rectangular waveguide, as illustrated in Fig. 1. Moreover, such dimensioned dielectric waveguides are the easiest to fabricate with a CNC machine, because no additional taper is needed. Besides, the rectangular shape maintains the polarization unlike circular waveguides. The fabricated dielectric waveguide has a measured loss of $4.5 \mathrm{~dB} / \mathrm{m}$ at $160 \mathrm{GHz}$ and the measured radiated loss is $2 \mathrm{~dB}$ for a bend radius of $2 \mathrm{~cm}$ with two $90^{\circ}$ arcs in a row.

Between the two waveguides a mode transformer, the second mentioned component, is needed. Instead of using a straight rectangular waveguide and a linear tapered horn with a sharp edge as proposed in [10], the shape of the mode transformer is optimized by numerical simulations. With the continuously opened horn and the enhancement at the dielectric waveguide taper, matching and insertion loss can be improved. Especially the tapered horn allows a smoother transition of the wave. Furthermore, the horn opening must be large enough that the field components in the air are stronger concentrated around the waveguide instead of scattered at the horn's metal edges. The fabricated mode transformer has a measured loss of $0.4 \mathrm{~dB}$.

\section{ElLiptical Dielectric Lens}

The synthesis of the elliptical lens is based on a geometrical optics approach [7], [11]. All the rays radiating from the point source have the same time delay when they reach the plane phase front as shown in Fig. 2. Therefore, the source must be in the focal point $c$ of the ellipse with one semi axis $a=R$ and the dielectric constant $\varepsilon_{r}$. With the geometrical optics conditions, the second semi axis $b$ is

$$
b=\frac{R}{\sqrt{1-1 / \varepsilon_{r}}}
$$

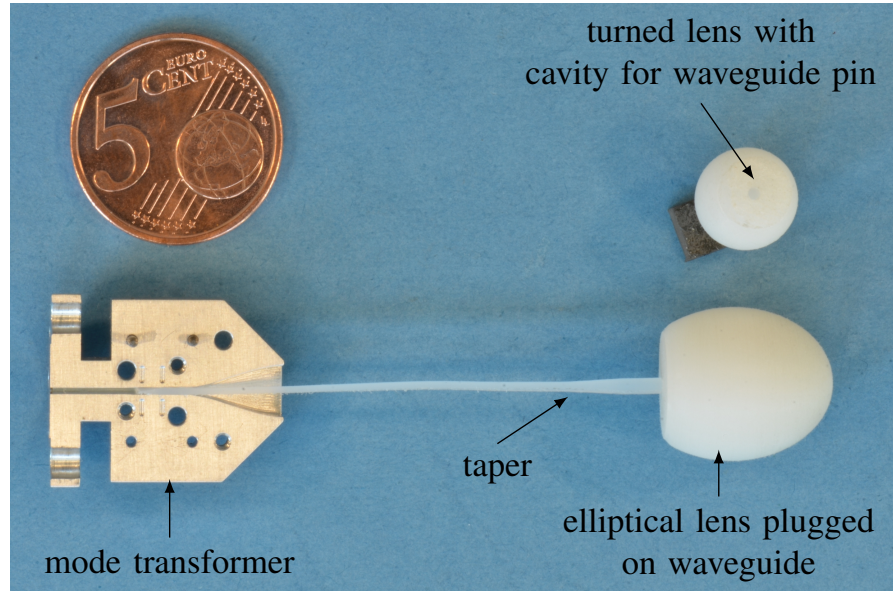

Fig. 3: Fabricated dielectric waveguide $(40 \mathrm{~mm})$, dielectric lenses and a mode transformer semi-monocoque.

and the focal point

$$
c=\frac{R}{\sqrt{\varepsilon_{r}-1}} .
$$

The ellipsoidal lens antenna then results from the rotation of the ellipse around the symmetry axis ( $x$-axis). The complete antenna with the feeding structure was simulated with a numerical transient solver so that the geometrical optics approach could be verified. Furthermore, different reflections from the lens surface, the mode transformer, and the transition from dielectric waveguide to lens are visible. The main reflection is caused by the transition from the dielectric waveguide to the lens and results from the impedance mismatch, because parts of the wave in the dielectric waveguide are in the air. To reduce this reflection, a taper to a larger rectangular cross section $(1295 \mu \mathrm{m} \times 1295 \mu \mathrm{m})$ is made to focus more field in the dielectric. However it is important that the rectangular cross section is not to large and the radius $R$ not to small that the geometrical-optical approach is valid.

Further matching improvement can be achieved by using a hemispherical lens shape or matching layers on the lens at the expense of additional fabrication steps.

The gain of the antenna depends on its size. In the typical order of the antenna dimension, the gain increases with increasing lens radius $R$.

\section{Measurement Results}

For the realization of the antenna, the different dielectric parts have to be connected to each other, because they could not be manufactured as one component.

At first the dielectric waveguide is fabricated from a $3 \mathrm{~mm}$ thick HDPE plate. The plate's thickness is reduced to the width of the waveguide, afterwards, the taper at the mode transformer and the taper before the antenna is milled with a $\mathrm{CNC}$ machine. At the end of the waveguide there is a pin of $2 \mathrm{~mm}$ length and a diameter of $1.2 \mathrm{~mm}$. This pin is stacked in a cavity at the centre of the elliptical antenna. This elliptical 


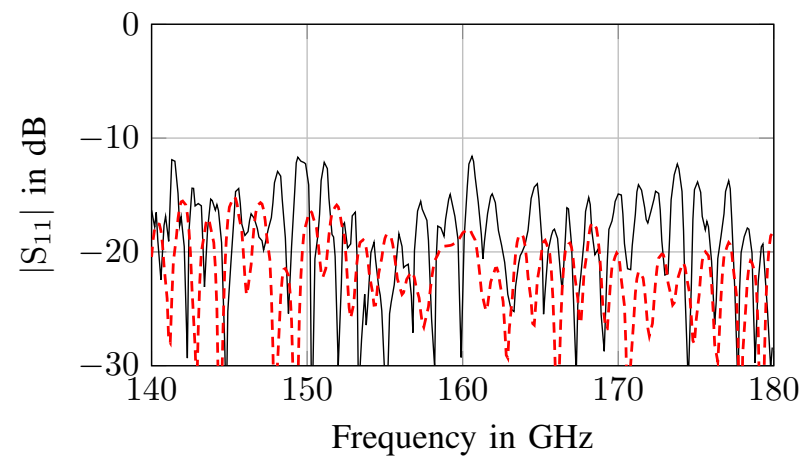

(a) Dielectric waveguide length $40 \mathrm{~mm}$.

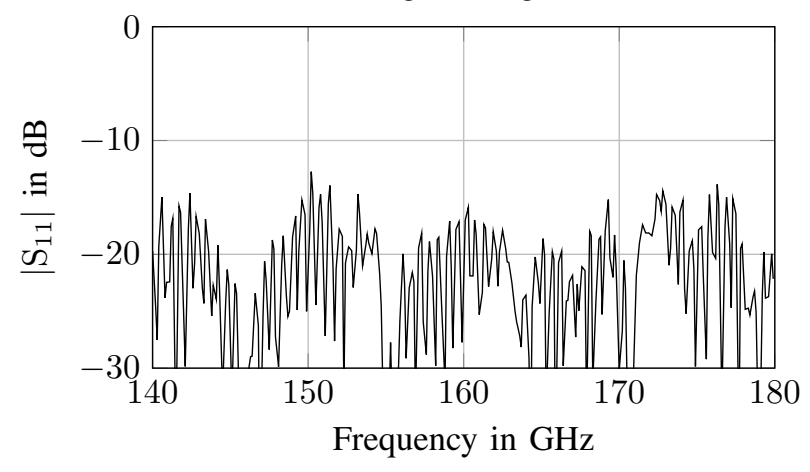

(b) Dielectric waveguide length $150 \mathrm{~mm}$.

Fig. 4: Matching of the elliptical antenna for different waveguide lengths (distance taper mode transformer to taper lens), measurement (-) and simulation (----).

antenna is fabricated from a HDPE plate as well and faced with a 5-axes CNC machine. Two antennas with different radii $R$, $5 \mathrm{~mm}$ and $7.5 \mathrm{~mm}$, were fabricated.

The mode transition is manufactured in semi-monocoque design from aluminium blocks. All the fabricated parts are illustrated in Fig. 3.

In the measurement setup used for the antenna characterisation, that is described in [12], the flexible antenna under test has to be fixed in a carrier made of Rohacell $\left(\varepsilon_{r} \approx 1\right)$.

\section{A. Matching}

The simulated and measured reflection coefficient of the antenna is illustrated in Fig. 4a. For the frequency range between $140 \mathrm{GHz}$ and $180 \mathrm{GHz}$ the measured $\mathrm{S}_{11}$ is below $-11.5 \mathrm{~dB}$. Compared to the simulation, the measured values are higher. The reason for this are air gaps in the plug connection between antenna and dielectric waveguide. This is also observable for the reflection coefficient for the longer waveguide in Fig. 4b. Here the periodicity in the ripples is higher, which results from the standing wave between antenna and mode transition. An inaccurate placement of the dielectric waveguide in the mode transformer and the roughness at the taper's surface increase the $\mathrm{S}_{11}$. The antenna's radii have no influence on the reflections, because the field is negligible at far distances $(>5 \mathrm{~mm})$ from the waveguide.

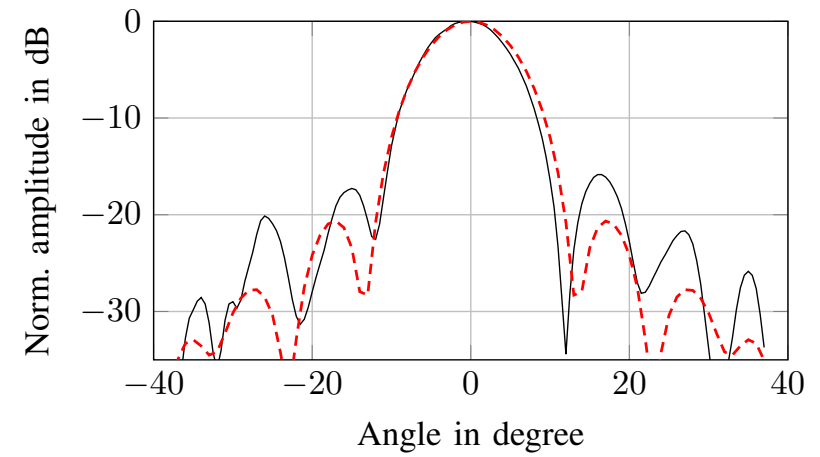

Fig. 5: Radiation pattern in the E-plane at $160 \mathrm{GHz}$ for $R=5 \mathrm{~mm}$, measurement ( - ) and simulation (----).

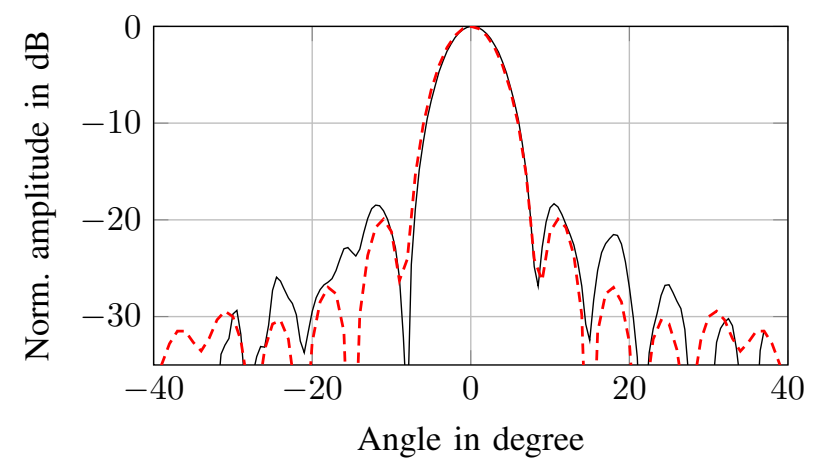

Fig. 6: Radiation pattern in the E-plane at $160 \mathrm{GHz}$ for $R=7.5 \mathrm{~mm}$, measurement $(-)$ and simulation (---).

\section{B. Radiation Pattern}

For the lens with radius $R=5 \mathrm{~mm}$ the radiation pattern at $160 \mathrm{GHz}$ in the E-plane is shown in Fig. 5. The $3 \mathrm{~dB}$ angular width is $10.2^{\circ}$ and the maximum sidelobe level is $-15.8 \mathrm{~dB}$. The measured results have just small deviations from the simulation with a sidelobe level of $-20.6 \mathrm{~dB}$.

The $3 \mathrm{~dB}$ angular width of the larger antenna with $R=7.5 \mathrm{~mm}$ amounts to $6.8^{\circ}$ for $160 \mathrm{GHz}$ as visible in Fig. 6 . Again the measurement agrees well with the simulation and the maximum side lobe level is with $-18.3 \mathrm{~dB}$ only $1.7 \mathrm{~dB}$ larger than the simulated one.

Due to the symmetry of the antenna, the H-plane pattern is very similar to the E-plane pattern and is consequently not depicted. Instead, an entire measured radiation pattern for the elevation angle from $-37^{\circ}$ to $37^{\circ}$ is shown in Fig. 7 . The data were measured for azimuth in $4^{\circ}$ steps and for elevation in $1^{\circ}$ steps. To smooth the plot, additional values were linear interpolated. The expected cylindrical symmetry is clearly visible.

The radiation patterns for other frequencies within the frequency range from $140 \mathrm{GHz}$ to $180 \mathrm{GHz}$ are very similar to the illustrated one. With increasing frequency, the $3 \mathrm{~dB}$ angular width decreases and the sidelobe level stays nearly unchanged. 


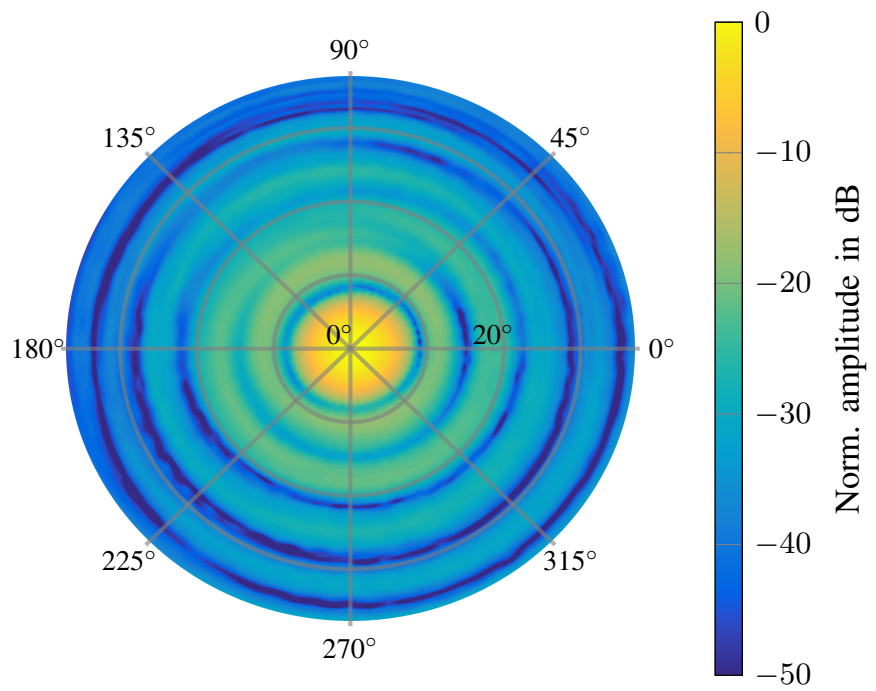

Fig. 7: Measured radiation pattern at $160 \mathrm{GHz}$ for $R=7.5 \mathrm{~mm}$.

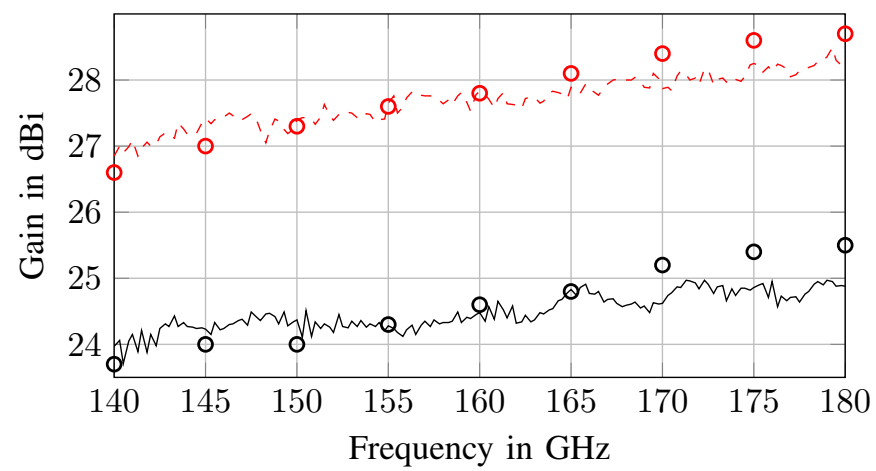

Fig. 8: Gain over frequency for the two different lens radii $R=5 \mathrm{~mm}$ (meas.: — (meas.: ----, sim.: o).

\section{Gain}

To measure the gain of the antenna, the determined losses from the mode transition and the dielectric waveguide were de-embedded from the measured values. For the measurement a $26 \mathrm{dBi}$ standard gain horn was used.

The gain as function of frequency for the smaller lens is depicted in Fig. 8. The gain is between $24 \mathrm{dBi}$ and $25 \mathrm{dBi}$. For the elliptical lens with radius $7.5 \mathrm{~mm}$ the gain is between $27 \mathrm{dBi}$ and $28.5 \mathrm{dBi}$. The gain increases with increasing frequency, since the wavelength is smaller compared to the lens size. The measured values for both antennas agree very well with the simulations.

\section{CONCLUSion}

In this paper, a flexible lens antenna with high gain and easy way to fabricate for the frequency range from $140 \mathrm{GHz}$ to $180 \mathrm{GHz}$ is presented. The flexibility of the antenna allows the use as a sniffer probe for a radar system.
The antenna is fed by a rectangular waveguide. Using a low-loss mode transition $(0.4 \mathrm{~dB})$, the wave travels over a flexible HDPE dielectric waveguide with losses of $4.5 \mathrm{~dB} / \mathrm{m}$ to an elliptical lens antenna, which is stacked on the dielectric waveguide. The lens was designed using a geometrical optics approach and for matching improvement a taper was designed with a full wave simulation. As a result, two antennas with a gain of $24.5 \mathrm{dBi}$ and $27.5 \mathrm{dBi}$ and a sidelobe level of $-15.8 \mathrm{~dB}$ and $-18.3 \mathrm{~dB}$, respectively, were measured.

\section{ACKNOWLEDGMENT}

This work was supported by the Ministry for Science, Research and Arts Baden-Württemberg within the project ZAFH MikroSens.

\section{REFERENCES}

1] E. A. J. Marcatili, "Dielectric Rectangular Waveguide and Directiona Coupler for Integrated Optics," The Bell System Technical Journal, vol. 48, no. 7, pp. 2071-2102, Sep. 1969.

2] J. Weinzierl, C. Fluhrer, and H. Brand, "Dielectric Waveguides at Submillimeter Wavelengths," in IEEE Sixth International Conference on Terahertz Electronics Proceedings, Sep. 1998, pp. 166-169.

[3] J. Weinzierl, J. Richter, G. Rehm, and H. Brand, "Simulation and Measurement of Dielectric Antennas at $150 \mathrm{GHz}$," in 29th European Microwave Conference, vol. 2, Oct. 1999, pp. 185-188.

[4] A. Rivera-Lavado, S. Preu, L. E. García-Muñoz, A. Generalov, J. Montero-de-Paz, G. Döhler, D. Lioubtchenko, M. Mèndez-Aller, F. Sedlmeir, M. Schneidereit, H. G. L. Schwefel, S. Malzer, D. SegoviaVargas, and A. V. Räisänen, "Dielectric Rod Waveguide Antenna as THz Emitter for Photomixing Devices," IEEE Transactions on Antennas and Propagation, vol. 63, no. 3, pp. 882-890, Mar. 2015.

[5] P. K. Verma, R. Kumar, and M. Singh, "Design and Simulation of Dielectric Tapered Rod as Feed for Dielectric Lens Antenna at $140 \mathrm{GHz}$," in International Conference on Recent Advances in Microwave Theory and Applications, Nov. 2008, pp. 233-235.

[6] A. Bisognin, D. Titz, C. Luxey, G. Jacquemod, F. Ferrero, D. Lugara, A. Bisognin, R. Pilard, F. Gianesello, D. Gloria, J. R. Costa, C. Laporte, H. Ezzeddine, E. B. Lima, and C. A. Fernandes, "A 120 GHz 3D-Printed Plastic Elliptical Lens Antenna with an IPD Patch Antenna Source,' in IEEE International Conference on Ultra-WideBand (ICUWB), Sep. 2014, pp. 171-174.

[7] N. Pohl, "A DIELECTRIC LENS ANTENNA WITH ENHANCED APERTURE EFFICIENCY FOR INDUSTRIAL RADAR APPLICATIONS," in IEEE Middle East Conference on Antennas and Propagation (MECAP), Oct. 2010, pp. 1-5.

[8] J. W. Lamb, "Miscellaneous data on materials for millimetre and submillimetre Optics," International Journal of Infrared and Millimeter Waves, vol. 17, no. 12, pp. 1997-2034, Dec. 1996.

[9] E. A. J. Marcatili, "Bends in Optical Dielectric Guides," The Bell System Technical Journal, vol. 48, no. 7, pp. 2103-2132, Sep. 1969.

[10] A. Hofmann, E. Hörster, J. Weinzierl, L.-P. Schmidt, and H. Brand, "Flexible Low-Loss Dielectric Waveguides for THz Frequencies with Transitions to Metal Waveguides," in 33rd European Microwave Conference, Oct. 2003, pp. 955-958.

[11] A. V. Boriskin, G. Godi, R. Sauleau, and A. I. Nosich, "Small Hemielliptic Dielectric Lens Antenna Analysis in 2-D: Boundary Integral Equations Versus Geometrical and Physical Optics,' IEEE Transactions on Antennas and Propagation, vol. 56, no. 2, pp. 485-492, Feb. 2008.

[12] L. Boehm, F. Boegelsack, M. Hitzler, and C. Waldschmidt, "An Automated Millimeter-Wave Antenna Measurement Setup Using a Robotic Arm," in IEEE International Symposium on Antennas and Propagation USNC/URSI National Radio Science Meeting, Jul. 2015, pp. 2109-2110. 